\title{
Transfer effects in monkey discrimination learning after extensive two-problem training
}

\author{
F. ROBERT TREICHLER ${ }^{1}$ \\ KENT STATE UNIVERSITY
}

Six monkeys were given 840 trials on each of two simple discriminations and then tested on 16, 20-trial problems using similar stimuli. The test performances indicated significant transfer from previous training with no significant inter- or intra-problem changes during testing. Further testing on concurrent discriminations showed slightly better performance with a common class of stimulus being correct in two problems than with different classes correct on the two problems if these conditions were used in alternate sessions. However, when only the latter procedure was used, Ss rapidly adopted solutions requiring discrimination of stimuli present on individual trials within a concurrent problem.

Several quantitative theoretical accounts (Restle, 1958; Levine, 1965) have attempted to describe response patterns which underlie the development of learningset performance. In each of these formulations the development of a "strategy," described as "win-stay, lose-shift with respect to object, " has been considered to be largely responsible for ultimately demonstrated performances.

An experiment by Schusterman (1962) has investigated the nature of training sufficient for the development of this strategy and has demonstrated that chimpanzees given extensive successive discrimination-reversal training on only three stimulus pairs exhibited a high level of proficiency (approximately $90 \%$ correct on Trial 2) immediately upon being tested on conventional learning-set problems.

The first phase of the present investigation was designed to determine whether rhesus monkeys given training on only two discrimination problems would develop a "strategy" and show transfer when tested on subsequent problems of limited trial length and similar stimulus characteristics. Because simple stimulus equivalence might yield transfer effects, later phases of testing under concurrent discrimination procedures were designed to investigate the nature of the response patterns adopted when correct solutions depended upon detecting specific properties of the discriminanda presented on individual trials.

\section{Methods and Results}

The Ss were six naive rhesus monkeys and testing was conducted in an automated WGTA adapted from one described by Meyer et al (1961). Stimuli were displayed on two $3 \times 4$ in. panels on the forward wall of a compartment. Display cells were capable of presenting any one of five flooded-field colors or seven geometric figures.

Trial sequences involved S placing its head in a form-fitting mask to initiate stimulus presentation on the cells. Touching a correct panel delivered a reinforcement to a cup beneath that panel and immediately extinguished stimuli and advanced the sequence to the next trial after a 2 sec. delay.

All problems utilized noncorrection techniques and involved simul- taneous presentation of a color on one panel and a form on the other. Four problem sequences were generated for use in each experiment and $\mathrm{S}$ assignments to these sequences were random for each problem. Sequence orders were randomly generated with the restriction that the number of possible reinforced trials was the same for each position, and, within each problem, stimuli were presented an equal number of times in each position.

Pretraining. Ss received 40 trials per day on either a triangle vs. green problem with color reinforced, or a circle vs. blue problem with form reinforced. One stimulus set was designated as the discrimination for a day, and all Ss received this problem. This was done for 42 days or 840 trials on each problem.

The proportion of correct responding gradually increased at about the same rate on both problems over the course of training. On the last presentation of each problem five animals were correct on all 40 trials and the one other $S$ was correct on 39 .

Twenty-trial Discrimination Learning. Sixteen new problems were constructed by pairing colors and forms from the stimulus set. Two 20-trial problems were presented each day and training was conducted for 8 days on 16 different problems.

Figure 1A displays the mean proportion of correct responses on Trials $2-20$ in successive four-problem blocks for each $\mathrm{S}$ and for all Ss combined. The vertical displacement of the function expressing performance of all Ss was significantly greater than chance $(p<.01)$ and all individual $S$ performances but $S 3$ were significantly different from chance (all p's $<.05 ; \mathrm{S} \mathrm{3,p}>.10$ ). Since interproblem improvement is a usual indication of learning-set acquisition, a test of linear trend over successive blocks was performed, but failed to yield significant results $(p>.10)$. Graphic plotting of mean proportion on the successive trials within problems indicated that near-asymptotic performance was obtained by about Trial 10. When separate plots for the four successive problem-blocks were compared, similar functions were obtained for each block and no systematic differences in intraproblem performance were evident over this segment of training.

Concurrent Discrimination: Concurrent discrimination tasks involving two intermixed 20-trial color vs. form problems were presented each day for the next 8 days. On Days $1,3,5$, and 7 , correct solutions involved the selection of the same kind of stimulus on both problems. On Days 2, 4,6, and 8, a color was reinforced on one problem while a form was reinforced on the other so that different classes of stimuli were correct in the two concurrent problems.

Since different types of solutions were required on alternate days, and, since this might have provided intersession interference, four additional days were run using only the conditions wherein a form and a color were reinforced in the concurrent set. Before this was begun, one animal died, our $\mathrm{N}$ was reduced to 5 , and a four-day delay was necessary for equipment modification. 


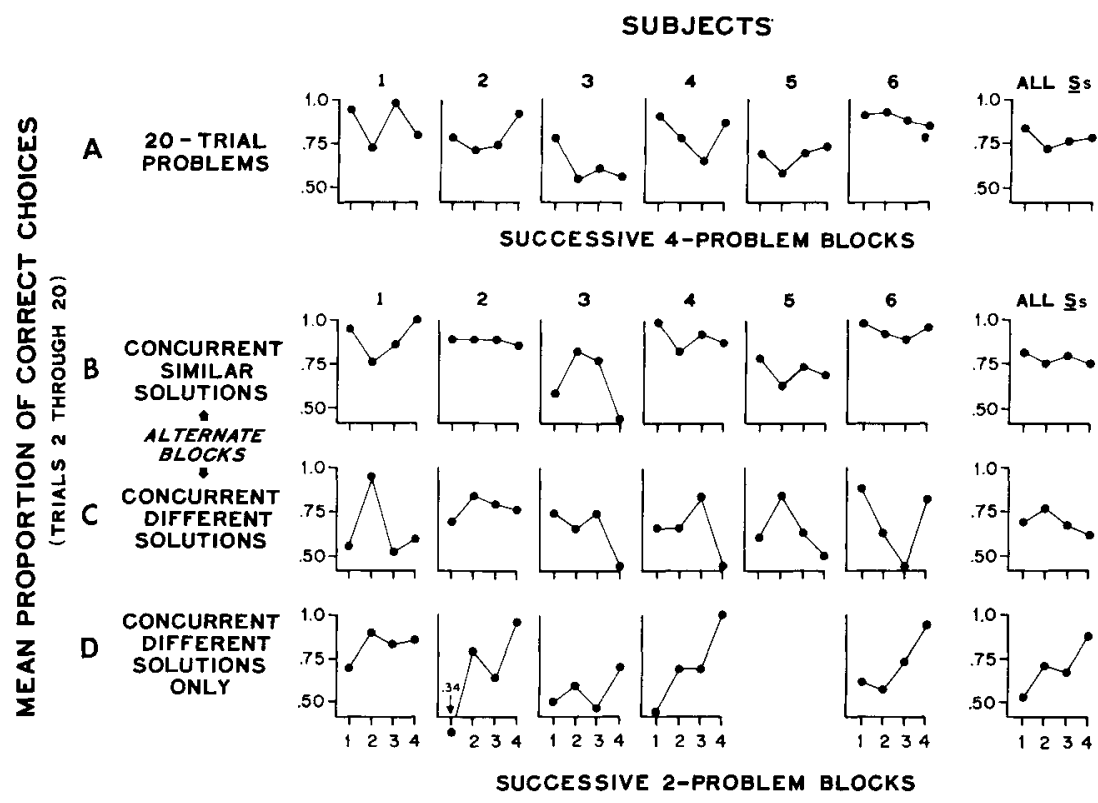

Fig. 1. Mean proportion correct on Trials 2 through 20 in the successive blocks under four discrimination testing procedures ( $A, B, C, D)$. Scores for individual Ss and means across all $\mathrm{Ss}$ are displayed.

Figure 1B shows the mean and individual proportions of correct choice on concurrent problems where solutions involved choosing the same class of stimulus (color or form) in both problems. Figure 1C displays the results from sessions where solutions depended upon choosing a form in one problem and a color in the other.

The vertical displacement of these functions indicates that performance in both cases was significantly better than chance $(B, p<.01 ; C, p<.01)$. Tests for linear trends through successive blocks failed to yield significance under either condition $(B, p>.10 ; C, p>.05)$ 。

A direct difference comparison between performance on concurrent problems with similar solutions and concurrent problems with different solutions indicated significantly greater $(p<.01)$ correct choice in the former condition.

Proportions of correct choices during the last four sessions are displayed in Fig, 1D. Here, too, overall performances were significantly greater than chance $(\mathrm{p}<.01)$, and, additionally, a significant linear trend $(p<.02)$ over successive blocks was observed.

\section{Discussion}

After the development of proficient performance on the two pretraining problems, immediate and persistent responding at better than chance levels was demonstrated in 16 subsequent 20-trial conventional discrimination problems. This suggests that some transfer effects do occur after training involving exposure to a limited set of simple discriminanda, but they do not result in the immediate adoption of win-stay, lose-shift strategies shown by Schusterman's (1962) chimpanzees.

It is possible that, because of the nature of the discriminations used in these experiments, a kind of stimulus equivalence of discriminanda or interproblem stimulus generalization was responsible for the observed transfer effect. The use of concurrent discrimination procedures was undertaken in order to give some indication of whether performances were different when solutions depended on the trial-by-trial use of specific stimulus properties as opposed to consistently choosing the same class of stimulus in two concurrent problems. These results indicated that the latter condition yielded better performance than the former. However, if interference effects were minimized, solutions that required responding on the basis of stimuli specific to a particular trial were readily adopted. It is concluded that, although generalized solutions may occur, monkeys tend to sample the stimuli present on discrete trials.

\section{References}

Levine, M. Hypothesis behavior. In A. M. Schrier, H. F. Harlow, and F. Stollnitz (Eds.), Behavior of nonhuman primates. Vol. I. New York: Academic Press, 1965. Pp. 97-127.

Meyer, D. R., Polidora, V. J., \& McConnell, D. G. Effects of spatial S-R contiguity and response delay upon discriminative performances by monkeys. J. comp. physiol. Psychol., 1964 $58,472-474$.

Restle, F. Toward a quantitative description of learning set data. Psychol. Rev., 1958, 65, 77-91.

Schusterman, R. J. Transfer effects of successive discriminationreversal training in chimpanzees. Science, 1962, 137, 422-423.

Note

1. This experiment was supported by funds obtained under Public Health Service grant MH-10335-01. 